ARTICLE

Received 27 Jul 2016 | Accepted 9 Mar 2017 | Published 8 May 2017

DOl: 10.1038/ncomms15194

OPEN

\title{
Atomic-level energy storage mechanism of cobalt hydroxide electrode for pseudocapacitors
}

Ting Deng ${ }^{1,2,3}$, Wei Zhang 1,2,3,4,5, Oier Arcelus ${ }^{4}$, Jin-Gyu Kim6, Javier Carrasco ${ }^{4}$, Seung Jo Yoo ${ }^{6}$, Weitao Zheng 1,2,3, Jiafu Wang1,2,3, Hongwei Tian ${ }^{1,2,3}$, Hengbin Zhang ${ }^{1,2,3}$, Xiaoqiang Cui ${ }^{1,2,3}$ \& Teófilo Rojo ${ }^{4,7}$

Cobalt hydroxide is a promising electrode material for supercapacitors due to the high capacitance and long cyclability. However, the energy storage/conversion mechanism of cobalt hydroxide is still vague at the atomic level. Here we shed light on how cobalt hydroxide functions as a supercapacitor electrode at operando conditions. We find that the high specific capacitance and long cycling life of cobalt hydroxide involve a complete modification of the electrode morphology, which is usually believed to be unfavourable but in fact has little influence on the performance. The conversion during the charge/discharge process is free of any massive structural evolution, but with some tiny shuffling or adjustments of atom/ion species. The results not only unravel that the potential of supercapacitors could heavily rely on the underlying structural similarities of switching phases but also pave the way for future material design for supercapacitors, batteries and hybrid devices.

\footnotetext{
${ }^{1}$ Department of Materials Science, Jilin University, Changchun 130012, China. ${ }^{2}$ Key Laboratory of Mobile Materials MOE, Jilin University, Changchun 130012 China. ${ }^{3}$ State Key Laboratory of Automotive Simulation and Control, Jilin University, Changchun 130025, China. ${ }^{4}$ CIC Energigune, 01510 Miñano, Spain. ${ }^{5}$ Ikerbasque, Basque Foundation for Science, 48013 Bilbao, Spain. ${ }^{6}$ Department of Electron Microscopy Research, Korea Basic Science Institute, Daejeon 34133, South Korea. ${ }^{7}$ Departamento de Química Inorgánica, Universidad del País Vasco, UPV/EHU, 48080 Bilbao, Spain. Correspondence and requests for materials should be addressed to W.Z. (email: weizhang@jlu.edu.cn) or to W.T.Z. (email: wtzheng@jlu.edu.cn).
} 
P seudocapacitance is the Faradaic charge transfer between the electrolyte and the (sub)surface of a suitable metal oxide/hydroxide electrode, involving reversible redox reactions, electroadsorption or intercalation processes ${ }^{1}$. Redox reaction is the main property in the functionality of supercapacitors $(\mathrm{SCs})^{2,3}$, but it also applies to a variety of other energy storage devices such as fuel cells ${ }^{4,5}$ and rechargeable batteries $^{6,7}$. Irreversible morphological changes during electrochemical cycles cause capacitance and cyclability degradations. So controlling morphological stability, therefore, is the common motif to unlock the potential of novel energy storage and conversion SC systems. Theoretically, in nickel-metal hydride batteries, the charge/discharge mechanism is based on the motion of $\mathrm{H}^{+}$species, the oxidation and reduction reactions can be interpreted as hydrogen ions switching through: $\mathrm{Ni}(\mathrm{OH})_{2}+\mathrm{OH}^{-} \leftarrow \rightarrow \mathrm{NiOOH}+\mathrm{H}_{2} \mathrm{O}+e^{-}$(refs 6,8,9). For a hydroxide-based supercapacitive electrode (for example, $\left.\mathrm{Co}(\mathrm{OH})_{2}\right)$, with high theoretical capacitance and long cycling life, it is well accepted that the charge/discharge mechanism is $\mathrm{Co}(\mathrm{OH})_{2}+\mathrm{OH}^{-} \leftarrow \rightarrow \mathrm{CoOOH}+\mathrm{H}_{2} \mathrm{O}+e^{-}$(refs 10,11). Both $\mathrm{Co}(\mathrm{OH})_{2}$ and $\mathrm{CoOOH}$ have the same layered cobalt structure and the large interlayer spacing that facilitates the intercalation of $\mathrm{H}^{+}$species. $\mathrm{Ni}(\mathrm{OH})_{2}$ exploited in nickel-metal hydride batteries shows a pair of well-separated Faradaic redox peaks, with a large voltage separation $(>0.1-0.2 \mathrm{~V})$ between oxidation and reduction due to a phase transition ${ }^{12}$. This indicates a fine battery behaviour according to the perspective of Simons et al. ${ }^{13} \cdot \mathrm{Co}(\mathrm{OH})_{2}$ shows a pair of redox peaks as well, however, the separation of these two peaks is vaguer and smaller, which indicates a so-called batterymimic mechanism. Provided with such a mechanism in a metal hydroxide electrode, the high specific pseudocapacitance and long cycling life of a SC and relevant structural transformations should be explained by a more fundamental motif rather than only morphological stability.

Here we show the energy storage/conversion mechanism of $\mathrm{Co}(\mathrm{OH})_{2}$ electrode, which can retain $95.7 \%$ of its initial capacitance after 8,000 cycles. Furthermore, along with in situ experimental examinations, the density functional theory (DFT) calculations provide more specific details of this mechanism, showing that the conversion during the charge/discharge procedure is free of any massive structural evolution, with only some tiny shuffling or adjustments of atom/ion species instead. According to our results, a battery-mimic mechanism at the atomic level can be applicable to pseudocapacitance, which may open an avenue in the design of new SC electrodes.

\section{Results}

Structural characterization of $\mathrm{Co}(\mathrm{OH})_{2}$. Figure 1a shows the $\mathrm{X}$-ray diffraction pattern of the as-prepared $\alpha-\mathrm{Co}(\mathrm{OH})_{2}$, and scanning electron microscopy (SEM) and transmission electron microscopy (TEM) analysis. The peak at $26^{\circ}$ belongs to the carbon fibre paper (CFP) substrate whose intensity is much weaker than the features of $\alpha-\mathrm{Co}(\mathrm{OH})_{2}$, indicating that $\alpha-\mathrm{Co}(\mathrm{OH})_{2}$ is well spread on the substrate. For more accurate information, we conducted synchrotron X-ray diffraction characterization (Supplementary Fig. 1), which confirms the peaks for (100) and (110), but also reveals the existence of a peak at $9.47^{\circ}(\lambda=1.24$ $\mathrm{nm}$ ) indexed as (003). The (003) peak is more intense and sharper than other peaks, indicating that $\alpha-\mathrm{Co}(\mathrm{OH})_{2}$ grows preferentially along the direction $<003\rangle$. In addition, Supplementary Fig. 1 also reveals the presence of nitrates into $\alpha-\mathrm{Co}(\mathrm{OH})_{2}$, which are incorporated during the synthesis. As shown in Fig. 1b,f, the active material forms an interlaced petallike morphology, with each petal composed of several $\alpha-\mathrm{Co}(\mathrm{OH})_{2}$ sheets. A variety of pore sizes inside those petals is typically believed to increase the number of adsorbed charge species. In a magnified high-resolution TEM image, the $\alpha-\mathrm{Co}(\mathrm{OH})_{2}(100)$ planes can clearly be identified (Fig. 1g, where ' $\mathrm{P}$ ' stands for porous regions). Such unique morphology facilitates redox reactions by shortening ion diffusion distance and, therefore, enhancing ionic conductivity, which is expected to yield a high specific pseudocapacitance. To be noted, we chose CFP as the substrate, not only because its chemical stability and the ability to shorten the distance of electron transport ${ }^{14}$ but also CFP can provide a robust bonding with $\mathrm{Co}(\mathrm{OH})_{2}$. We examined the surface state of the interface before and after the electrodeposition of $\mathrm{Co}(\mathrm{OH})_{2}$ (Supplementary Fig. 2), and we can see that a strong interaction was yielded between the substrate and the active material. The $\mathrm{Co}(\mathrm{OH})_{2}$ synthesized on $\mathrm{Ni}$ foam (Supplementary Fig. 3) with a higher capacitance compared to $\mathrm{Co}(\mathrm{OH})_{2}$ on CFP, however, shows a very poor cyclability (only $71.4 \%$ capacitance retention after 1,000 cycles). So another advantage of CFP is the strong interaction between CFP and $\mathrm{Co}(\mathrm{OH})_{2}$, which prevents the active material from being peeling off, thus leading to a stable platform to study the intrinsic properties of $\mathrm{Co}(\mathrm{OH})_{2}$.

However, it is important to notice that the synthesis of $\alpha-\mathrm{Co}(\mathrm{OH})_{2}$ generally results in poor crystalline and disordered structures ${ }^{15-17}$, which can rapidly transform into the more stable $\beta$-phase in alkaline media ${ }^{18}$. Therefore, before conducting electrochemical experiments, we settled the electrode in the electrolyte ( $\mathrm{KOH}$ solution) for $15 \mathrm{~min}$ to make $\mathrm{OH}^{-}$infiltrate into the pores to fully impregnate $\mathrm{Co}(\mathrm{OH})_{2}$ (allow ions permeate into electrodes and make full use of active materials). After bathed in the electrolyte, we observed a concomitant, most spectacular morphological change (Fig. 1c), where the loosestacked interlaced petals transformed into regular hexagonal platelets (the same phenomenon was also found in the residuals). This suggests that $\alpha-\mathrm{Co}(\mathrm{OH})_{2}$ interacts with hydroxide like a scalpel that removes the interlayer nitrates. According to Fourier transform infrared spectra (Supplementary Fig. 4a), an intense absorption band at $1,384 \mathrm{~cm}^{-1}$ is due to $\mathrm{N}-\mathrm{O}$ stretching vibration of $\mathrm{NO}_{3}^{-}$in the case of the as-prepared $\mathrm{Co}(\mathrm{OH})_{2}$. The relative intensity and the integral area of the peak reduced (Supplementary Fig. 4b) after bathing in $\mathrm{KOH}$ for $15 \mathrm{~min}$, indicating the removal of most $\mathrm{NO}_{3}^{-}$species. However, the $\mathrm{NO}_{3}^{-}$ions in inner layers of $\mathrm{Co}(\mathrm{OH})_{2}$ cannot be removed immediately, resulting in the remain of the peak. The wide band at $3,473 \mathrm{~cm}^{-1}$ is assigned to the $\mathrm{O}-\mathrm{H}$ stretching modes of interlayer water and of $\mathrm{H}$-bound $\mathrm{OH}$ group. After bathing, a sharp and intense band appeared at $3,629 \mathrm{~cm}^{-1}$, which is characteristic of free $\mathrm{OH}$ groups in $\beta$-type $\mathrm{Co}(\mathrm{OH})_{2}$. And other bands are due to $\mathrm{Co}-\mathrm{O}$ stretching and $\mathrm{Co}-\mathrm{OH}$ bending vibrations. As a result, the layers become more compact, with an ultimate hexagonal shape and presenting various thicknesses. Such drastic change is also reflected by the X-ray diffraction pattern shown in Fig. 1a, which indicates that the actual reactant in the alkaline environment was $\beta-\mathrm{Co}(\mathrm{OH})_{2}$. In addition, our DFT calculations indicate that the low-index hexagonal (0001) surface (Supplementary Fig. 5) is among the three lowest-energy surfaces of $\beta-\mathrm{Co}(\mathrm{OH})_{2}$ (Supplementary Table 1), which is consistent with the observed regular hexagonal platelets.

The change of specific surface area after this morphology modification was examined via $\mathrm{N}_{2}$ adsorption $\left(\mathrm{N}_{2}\right.$ isotherms in Supplementary Fig. 6). The Brunauer-Emmett-Teller (BET) surface area of the as-prepared $\mathrm{Co}(\mathrm{OH})_{2}$ was found to be $56.220 \mathrm{~m}^{2} \mathrm{~g}^{-1}$. After the modification, BET surface area increased to $110.280 \mathrm{~m}^{2} \mathrm{~g}^{-1}$. The appearance of hexagonal $\mathrm{Co}(\mathrm{OH})_{2}$ platelets and the corresponding activation of some pores are responsible for the specific surface area increase, which is favourable for the redox reaction. However, the limited increase of BET surface area is still too low to play a significant role in 
enhanced electrochemical performance. Indeed, we note that an increase of the BET surface area does not necessarily involve an increase of the specific capacitance, as shown by Ghodbane et al. ${ }^{19}$ These authors prepared a series of $\mathrm{MnO}_{2}$ allotropic phases with different BET surface areas, and the specific capacitance measured for each $\mathrm{MnO}_{2}$ phase did not follow the trend of the BET surface area. The contact of electrode and electrolyte is inevitable, and the coincidence is that the species that make $\mathrm{Co}(\mathrm{OH})_{2}$ ready for electrochemical tests is just the electrolyte ( $\mathrm{KOH}$ solution) itself. The management of military transformation instead of its civilian
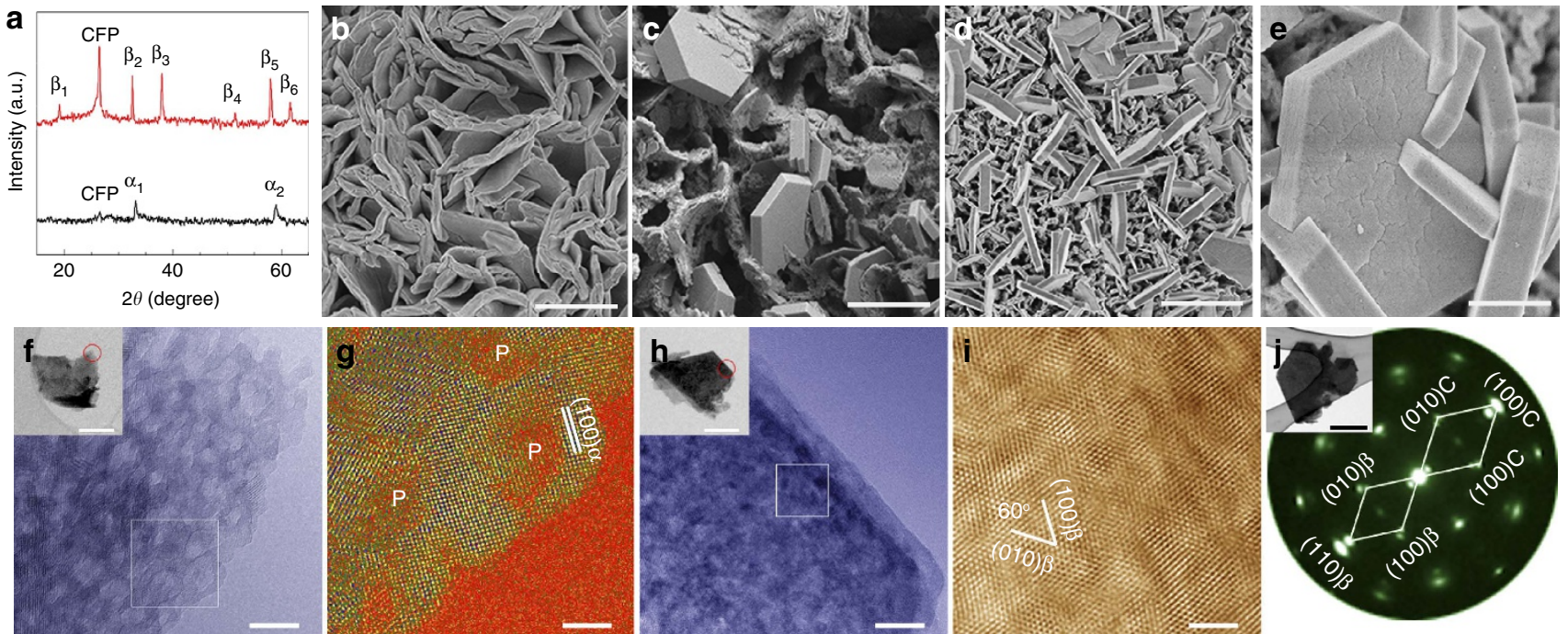

Figure 1 | Characterization of $\mathbf{C o}(\mathbf{O H})_{2}$ before and after in situ XAS experiment. (a) X-ray diffraction patterns of as-prepared $\mathrm{Co}(\mathrm{OH})_{2}, \alpha_{1}$ and $\alpha_{2}$ are indexed to $\alpha-\mathrm{Co}(\mathrm{OH})_{2}(100)$ and (110) (PDF, card No. 46-0605), and $\mathrm{Co}(\mathrm{OH})_{2}$ after bathing in $1 \mathrm{M} \mathrm{KOH}$ for 15 min, $\beta_{1}-\beta_{6}$ are indexed to (001), (100), (101), (102), (110) and (111) (PDF card No. 30-0443), respectively. (b-e) SEM images of as-prepared $\mathrm{Co}(\mathrm{OH})_{2}, \mathrm{Co}(\mathrm{OH})_{2}$ after bathing in $\mathrm{KOH}$ and after in situ XAS. Scale bars, $2 \mu \mathrm{m}, 2 \mu \mathrm{m}, 2 \mu \mathrm{m}$ and $400 \mathrm{~nm}$, respectively. (f-j) TEM images of $\mathrm{Co}(\mathrm{OH})_{2}$ and $\mathrm{Co}(\mathrm{OH})_{2}$ after in situ XAS. (f,h) High-resolution TEM (HRTEM) images of the areas marked by the red circles in the insets. Scale bars, $10 \mathrm{~nm}$ and the inset scale bars, $200 \mathrm{~nm}$. (g,i) HRTEM images that are highlighted by a white square in $\mathbf{f}, \mathbf{h}$, respectively. Scale bars, $3 \mathrm{~nm}$. (j) A local selected area electron diffraction pattern of $\mathrm{Co}(\mathrm{OH})_{2}$ after in situ XAS. The inset scale bar, $0.5 \mathrm{~mm}$.

a

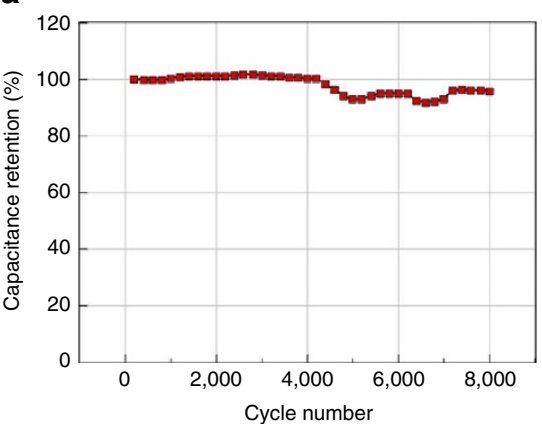

C

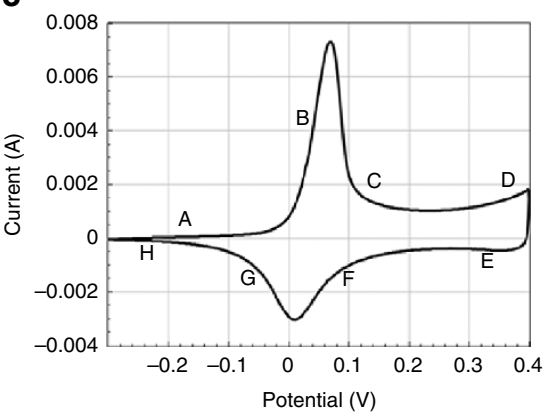

b

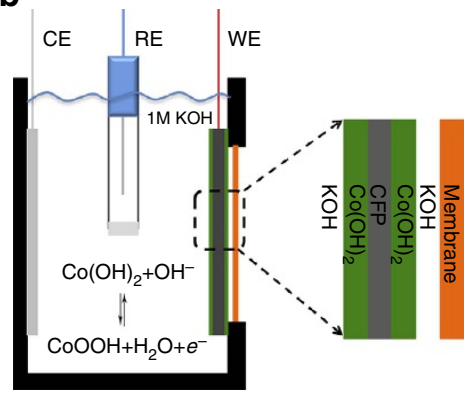

d

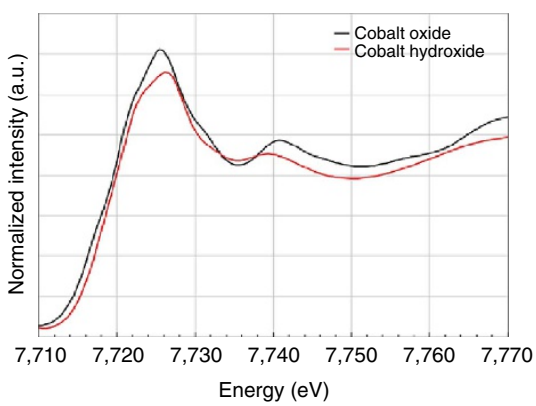

Figure 2 | Cycling life of $\mathbf{C o}(\mathbf{O H})_{2}$ and design of the XAS experiment. (a) The synthesized $\mathrm{Co}(\mathrm{OH})_{2}$ shows excellent cyclic stability, and the pseudocapacitance retention reaches $95.7 \%$ of its initial value after 8,000 cycles. (b) The design of the reaction cell for in situ XAS experiment. Within the cell, a three-electrode system is utilized, WE, CE and RE stand for working, counter and reference electrodes, respectively. (c) The in situ cyclic voltammetry curve of $\mathrm{Co}(\mathrm{OH})_{2}$ in the reaction cell and the scan rate is $0.4 \mathrm{mVs}^{-1}$. A-H labels correspond to the sampling points of XAS. (d) Comparison of XANES data collected on as-prepared $\mathrm{Co}(\mathrm{OH})_{2}$ electrode and $\mathrm{CoO}$ standard sample. 
counterpart at the level of the $\mathrm{Co}(\mathrm{OH})_{2}$ unit cell shows higher crystallinity according to the X-ray diffraction patterns, which indicates better conductivity of $\mathrm{Co}(\mathrm{OH})_{2}$.

Electrochemical performance evaluation. Supplementary Fig. 7 show cyclic voltammetry curves of $\mathrm{Co}(\mathrm{OH})_{2}$ and galvanostatic charge/discharge curve in $1 \mathrm{M} \mathrm{KOH}$ solution, respectively. The existence of a pair of redox peaks indicates a one-electrontransfer process and confirms that the pseudocapacitance behaviour is rooted in a redox reaction. During charge, $\mathrm{Co}(\mathrm{OH})_{2}$ is oxidized to $\mathrm{CoOOH}$, whereas in discharge the reaction is reversed. To be noted, there is a second redox process that has been discussed in many works: $\mathrm{CoOOH}+\mathrm{OH}^{-} \leftarrow \rightarrow \mathrm{CoO}_{2}+$ $\mathrm{H}_{2} \mathrm{O}+\mathrm{e}^{-}$. The second process is related to many factors, such as specific surface area ${ }^{10,20}$. The interlayer distance between adjacent $\mathrm{Co}(\mathrm{OH})_{2}$ single sheets can also influence the redox reaction, which occurs on the interface between electrolyte and active materials ${ }^{21}$. In our work, in addition to the low specific surface area of $\mathrm{Co}(\mathrm{OH})_{2}$ we synthesized, the decreased interlayer spacing due to the loss of $\mathrm{NO}_{3}^{-}$species hinders the complete penetration of the electrolyte. Thus, the oxidation process to $\mathrm{CoOOH}$ is expected to dominate. According to X-ray absorption spectroscopy (XAS) sampling that is statistically averaging, and the following results of in situ XAS experiments, the second process was incomplete and can be neglected when compared to the first reaction. However, under these circumstances, $\mathrm{Co}(\mathrm{OH})_{2}$ still exhibited great performance, which pales the role of interlayer spacing, nominal BET surface and some other factors in activating this performance. Surprisingly, there is no significant capacity loss after 8,000 cycles ( $95.7 \%$ capacity retention) as shown in Fig. 2a. The pathways for ion transport and diffusion to inner layer of $\mathrm{Co}(\mathrm{OH})_{2}$ could be forged and opened, which is responsible for capacitance increase after 5,000 and 7,000 cycles. The difference of peak currents is caused by semiconductor nature and aggregation of large mass. When the mass gets lower, the same phenomenon is also presented (Supplementary Fig. 8) with better electrochemical properties (high specific capacitance, excellent rate capability and long cycling life) (Supplementary Fig. 9). A unit of $800 \mathrm{Fg}^{-1}$ (at the current density of $2 \mathrm{Ag}^{-1}$ ) was achieved, which can be calculated according to the equation ${ }^{22}: C_{m}=C / m=(I \times \Delta t) /(\Delta V \times m)$, where $C_{m}\left(\mathrm{Fg}^{-1}\right)$ is the specific capacitance, $I(\mathrm{~mA})$ the discharge current, $\Delta t(\mathrm{~s})$ the discharging time, $\Delta V(\mathrm{~V})$ represents the potential drop during discharge process and $m$ (mg) the mass of the $\mathrm{Co}(\mathrm{OH})_{2}$. Accordingly, 756, 654 and $640 \mathrm{Fg}^{-1}$ was also yielded at the current density of 4,8 and $16 \mathrm{Ag}^{-1}$, respectively. Even at the current density of $32 \mathrm{Ag}^{-1}$, a capacitance of $523 \mathrm{Fg}^{-1}$ was obtained, which proves the good rate capability of $\mathrm{Co}(\mathrm{OH})_{2}$. And an asymmetric $\mathrm{Co}(\mathrm{OH})_{2}$-active carbon capacitor was fabricated and showed excellent performance (the capacitance reaches $71 \mathrm{Fg}^{-1}$ at the current density of $1 \mathrm{Ag}^{-1}$ and the energy density of the asymmetric capacitor can be calculated as $20.74 \mathrm{Wh} \mathrm{kg}^{-1}$ at a power density of $1,450 \mathrm{~W} \mathrm{~kg}^{-1}$; Supplementary Fig. 10). Nevertheless, a large mass is a prerequisite for acquiring good XAS signals in $\mathrm{KOH}$ aqueous solution, where X-ray intensities decay. For comparison, XAS measurements were also performed on the as-prepared electrode, the electrode after in situ experiment, as well as $\mathrm{CoO}$ as reference. Operated in a self-designed electrolytic cell (Fig. 2b, see the photographs of the device in Supplementary Fig. 11), the cyclic voltammetry curve shows no obvious difference (Fig. 2c).

In situ atomic level mechanism analysis. We chose in situ XAS as a powerful tool to qualitatively and quantitatively analyse how a

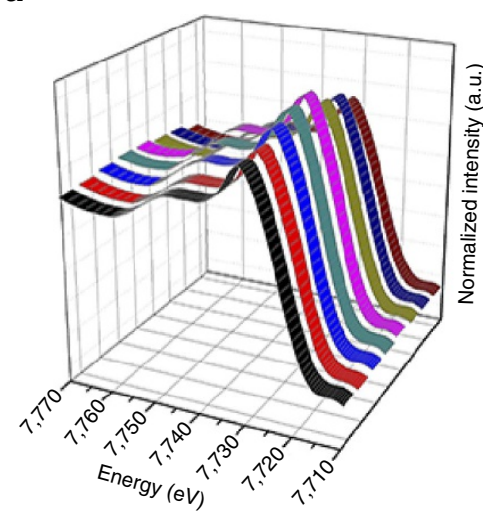

C

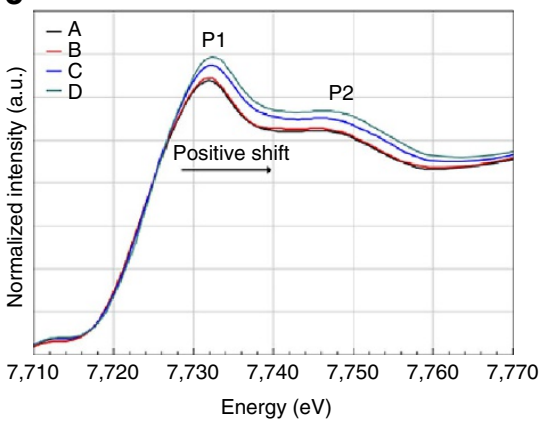

b

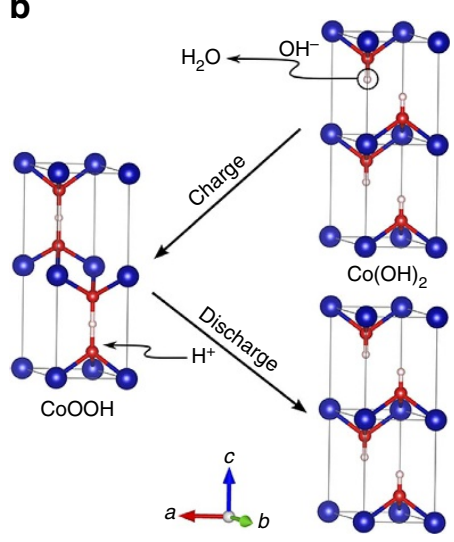

d

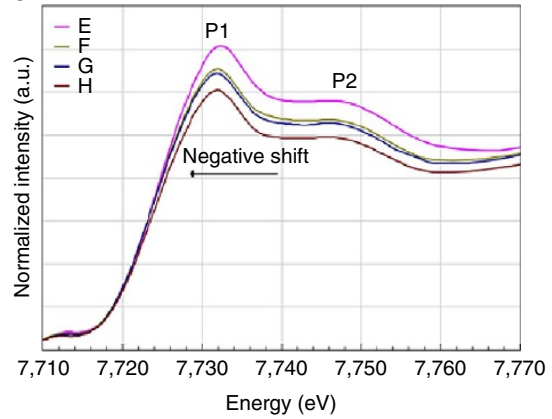

Figure 3 | Comparison of in situ XANES collected on the electrode and the reaction model of $\mathrm{Co}(\mathrm{OH})_{2}$ and $\mathrm{CoOOH}$ transformation. (a) In situ XANES spectra of a whole charge/discharge cycle in a three-dimensional mode. A-H spectra correspond to the positions marked in Fig. 2c. (b) The reaction model of $\mathrm{Co}(\mathrm{OH})_{2}$ and $\mathrm{CoOOH}$ phase transformation. $\mathrm{Co}(\mathrm{OH})_{2}$ loses a hydrogen to becomes $\mathrm{CoOOH}$ during charge, and vice versa, a hydrogen is incorporated into $\mathrm{CoOOH}$ during discharge. (c) In situ XANES spectra of the charge process. (d) In situ XANES spectra of the discharge process. 
the structural changes occur at atomic level. Thus, we can find out what actually controls the morphology to be stable and thus conclude how to minimize the influence of morphological change in actual operations. Figure 2d shows the X-ray absorption nearedge structure (XANES), the absorption K-edge for as-prepared $\mathrm{Co}(\mathrm{OH})_{2}$ is quite close to that of $\mathrm{CoO}$, suggesting that the valence of cobalt is +2 . (The $\mathrm{CoO}$, purchased from Alfa Aesar, may contain up to $10 \% \mathrm{Co}_{3} \mathrm{O}_{4}$, which is noted in the label. The existence of $\mathrm{Co}_{3} \mathrm{O}_{4}$ with higher valence state makes some positive shifting of XANES for $\mathrm{CoO}$ when compared to pure $\mathrm{CoO}$.) However, the spectrum of the electrode after 30 cycles shows a shift in the edge position to a higher energy (Supplementary Fig. 12). As mentioned above, thick layers (large mass) of $\mathrm{Co}(\mathrm{OH})_{2}$ is key to acquire good XAS signals. But this large mass resulted in conductivity decrease, which certainly would reduce the reversibility of the transformation of $\mathrm{Co}(\mathrm{OH})_{2}$ and $\mathrm{CoOOH}$. So some $\mathrm{CoOOH}$ remained and led to positive shift in Supplementary Fig. 12. In Fig. 1h, no obvious pores can be observed. $\beta-\mathrm{Co}(\mathrm{OH})_{2}$ can be well identified, as indicated by the (100) and (010) crystal planes with an acute angle $60^{\circ}$ (Fig. 1i). According to the comparison of the spectra, Co element presents two valences indicating the co-existence of $\mathrm{Co}(\mathrm{OH})_{2}$ and $\mathrm{CoOOH}$, which is also in accordance with the cyclic voltammetry curve and proved by selected area electron diffraction pattern (Fig. 1j), where $\beta-\mathrm{Co}(\mathrm{OH})_{2}$ and $\mathrm{CoOOH}$ (denoted as $\mathrm{C}$ ) exist coherently with an orientation relationship of $(100) \beta / /(100) C$, $(010) \beta / /(010) \mathrm{C}, \quad<001>\beta / /<001>C$. The XANES spectra collected in Fig. 3a summarizes the overall charge/discharge process, which is schematically represented in Fig. 3b. In addition, Fig. $3 c, d$ separately compares the in situ XANES spectra for the charge and discharge process, respectively. The absorption K-edges in Fig. 3c show a gradual positive shift while the potential increases from -0.3 to $0.4 \mathrm{~V}$ (Supplementary Table 2). This proves that the Co valence increases during the charge process ${ }^{23}$. The P1 peak gains intensity during the charge process due to a decrease in the local disorder of the nearest neighbours; the P2 peak also gains intensity, which indicates a stronger $\mathrm{Co}-\mathrm{Co}$ shell. Consistently, we observe a negative shift and intensity decrease of P1 and P2 peaks during discharge (Fig. 3d). This evidences the gradual valence decrease of Co atoms. Interestingly, spectra A and $\mathrm{H}$ show almost the same absorption K-edge positions, demonstrating the valence circulation. However, no other features can be observed in the oscillation pattern, which suggests the absence of a major structural change.

To gain more insight into the proposed structural models shown in Fig. 3b, we carried out a series of DFT calculations. Figure 4a shows the computed energy profile for the $\mathrm{Co}(\mathrm{OH})_{2} \rightarrow$ $\mathrm{CoOOH}$ phase transformation. We first considered the removal of one $\mathrm{H}$ atom per formula unit from $\mathrm{Co}(\mathrm{OH})_{2}$ to form $\mathrm{CoOOH}$, using the DFT-optimized lattice constants of $\mathrm{Co}(\mathrm{OH})_{2}$ $(a=b=3.176 \AA ; c=9.358 \AA)$. We found that a structure with one $\mathrm{H}$ atom per $\mathrm{CoO}$ layer (state $\mathrm{A}$ ) is $571 \mathrm{meV}$ more stable than a system that alternates fully hydrogenated $\mathrm{CoO}$ layers with empty ones (state B). We then examined the evolution of structures $\mathrm{A}$ and $\mathrm{B}$ into the $\mathrm{CoOOH}$ ground state (state $\mathrm{C}^{\star}$ ), which involves the concerted migration of $\mathrm{O}$ and/or $\mathrm{OH}$ species. In addition, we considered two different scenarios regarding the imposed lattice constants of the cell during the phase transformation. In the left side of the energy profile in Fig. 4a, we fixed the lattice constants to those of the DFT-optimized $\mathrm{Co}(\mathrm{OH})_{2}$, whereas in the right side we considered the lattice constants of the $\mathrm{CoOOH}$ ground state $(a=b=3.036 \AA ; c=8.862 \AA)$. In both cases we found that the lowest transition state structures ( $\mathrm{T} 1$ and $\mathrm{T}^{\star}$, respectively) correspond to the simultaneous diffusion of one $\mathrm{O}$ atom and one $\mathrm{OH}$ group within each $\mathrm{CoO}$ layer. $\mathrm{T}^{\star}$ transition state is only $44 \mathrm{meV}$ lower in energy than the T1, which indicates that $\mathrm{Co}(\mathrm{OH})_{2} \leftrightarrow \mathrm{CoOOH}$ lattice relaxation plays a minor role in the stabilization of the transition state structures. Overall, our DFT results suggest that the structural transformation towards $\mathrm{CoOOH}$ on $\mathrm{Co}(\mathrm{OH})_{2}$ deprotonation involves the rearrangement of both $\mathrm{O}$ atoms and $\mathrm{OH}$ groups, with lattice relaxation affecting mainly to the stabilization of the $\mathrm{CoOOH}$ ground state.

In situ extended X-ray absorption fine structure (EXAFS) spectra showing similar patterns were recorded. This implies no much difference of the coordination environment (Fig. 4b). P3 and $\mathrm{P} 4$ in the EXAFS curves correspond to the $\mathrm{Co}-\mathrm{O}$ and $\mathrm{Co}-\mathrm{Co}$ shells ${ }^{24}$. When the charge process is proceeding, both peaks have a gradual negative shift (Supplementary Table 6), indicative of the decreasing distance of $\mathrm{Co}-\mathrm{O}$ and $\mathrm{Co}-\mathrm{Co}$. The increasing intensities of both peaks show that structural disorder is reduced while the general structure is maintained (Fig. 4c). The entirely opposite situation occurs during the discharge process. Since the phase transformation is continually changing during the redox reaction, we thereof fit the spectra as three spectra (A, D and $H)$. The fitted curves are shown in Fig. $4 \mathrm{e}-\mathrm{g}$, and the fitting results are listed in Supplementary Table 7. The coordination numbers $(N)$ of $\mathrm{Co}-\mathrm{O}$ and $\mathrm{Co}-\mathrm{Co}$ are lower than theoretical values, which indicate phase distortions, the presence of non-diffraction, small particle domain and also the presence of a minority of phase. The variation trend of $\mathrm{Co}-\mathrm{O}$ and $\mathrm{Co}-\mathrm{Co}$ bond lengths $(R)$ is consistent with in situ EXAFS spectra. $R$ values of $\mathrm{Co}-\mathrm{O}$ and $\mathrm{Co}-\mathrm{Co}$ decrease for the charge process while increase on discharging. $\mathrm{Co}-\mathrm{O} / \mathrm{Co}-\mathrm{Co}$ bond lengths vary within $0.06 / 0.22 \AA$ during each charge/discharge cycle, which indicate the feasibility of this transformation. In addition, our DFT results indicate that the energy difference between the directly deprotonated $\mathrm{Co}(\mathrm{OH})_{2}$ and ground-state $\mathrm{CoOOH}$ structures (states $\mathrm{A}$ and $\mathrm{C}^{*}$ in Fig. $4 \mathrm{a}$, respectively) are separated by only $0.59 \mathrm{eV}$, with an activated energy barrier as low as $0.76 \mathrm{eV}(1.34 \mathrm{eV})$ for the forward (backward) process (transition state $\mathrm{T} 1^{*}$ in Fig. 4a). These results lead us to correlate excellent pseudocapacitance properties with the structural similarity of $\mathrm{Co}(\mathrm{OH})_{2}$ and $\mathrm{CoOOH}$, in which massive atom rearrangements are unnecessary for this mutual redox transformation, and thus save much energy from phase transition and enhance columbic efficiency. $\mathrm{Co}(\mathrm{OH})_{2}$ and $\mathrm{CoOOH}$ delicately adjust themselves to transform mutually into each other. The merit offers this reaction with a rapid switching rate, thus contributes to high power density, excellent rate capability and long cycling life. So to completely understand the reaction process, we chose in situ XAS to qualitatively and quantitatively study the structure transformation. To be complementary, we showed the SEM images before and after in situ XAS experiments. The hexagonal platelets in Fig. 1d,e show more quantity, more regularity, better homogeneousness and more uniform sizes than that of the $\mathrm{Co}(\mathrm{OH})_{2}$, in Fig. 1c, which spontaneously transformed from the as-prepared $\mathrm{Co}(\mathrm{OH})_{2}$. This is attributed to the electrochemical process. The bathed $\mathrm{Co}(\mathrm{OH})_{2}$, which may not be so regularly transformed from $\alpha$-type, were gradually trimmed to be more regular under potential loading after an entire electrochemical cycle. Another key observation is that the platelets were uniformly distributed on the substrate with a minority of small scratches on the surface. This is the evidence of volume expansion and contraction. In contrast with the pristine porous structure, a more compacted, layered structure was formed consisting of a majority of $\beta-\mathrm{Co}(\mathrm{OH})_{2}$ (Fig. 1h,i).

For a better understanding of the relation between structural similarity and the redox process, it is interesting to consider the iron-series oxides and hydroxides. These three transition metals share similar electronic configurations $\left(3 d^{6} 4 s^{2}, 3 d^{7} 4 s^{2}\right.$ and $3 d^{8} 4 s^{2}$ ), but exhibit quite different pseudocapacitance properties. For example, $\mathrm{Ni}(\mathrm{OH})_{2}$ has a large pseudocapacitance ${ }^{9,25}$, holding 
a
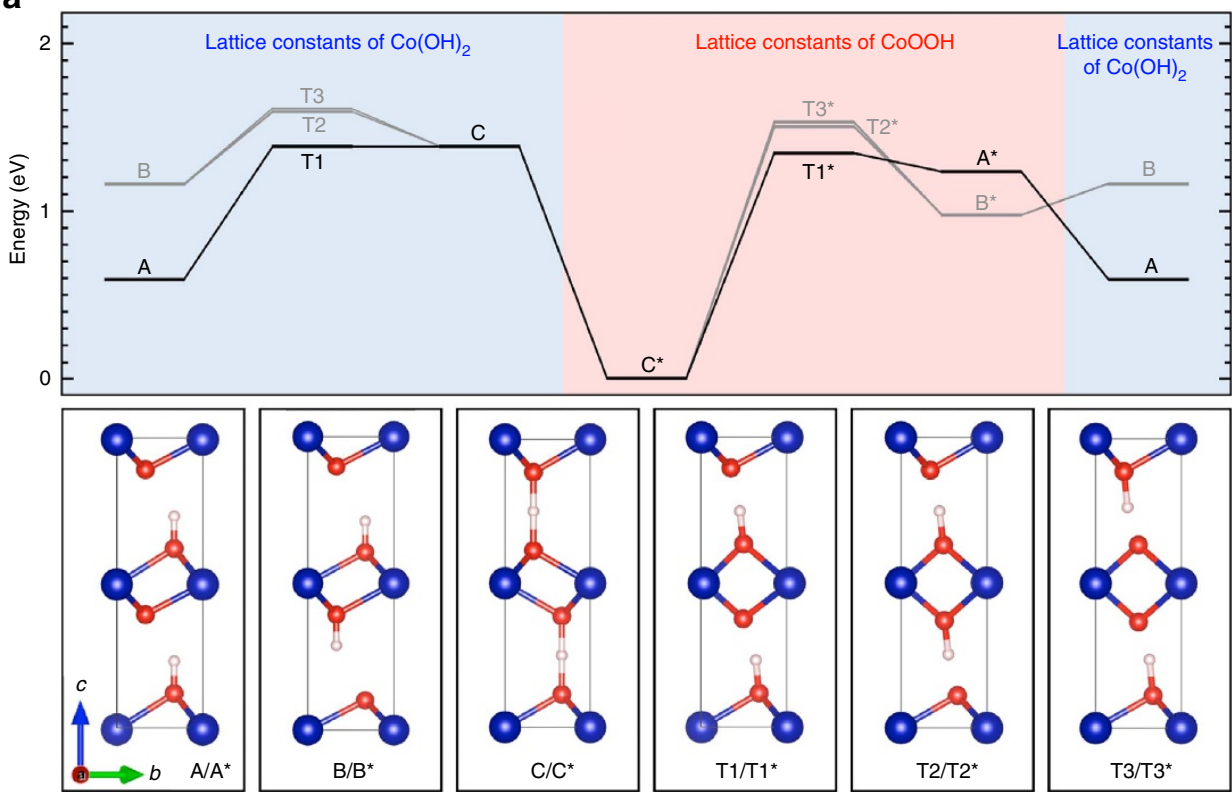

b

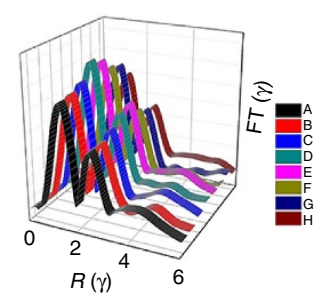

e

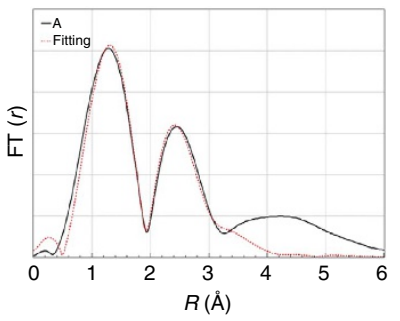

C

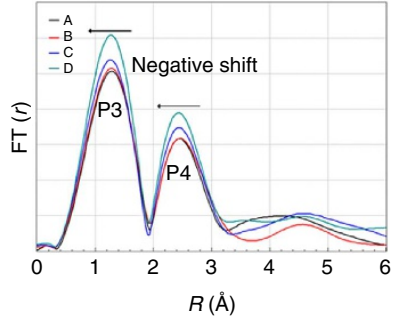

f

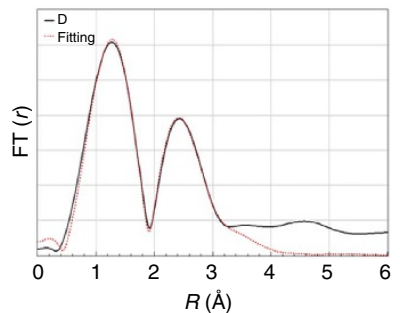

d

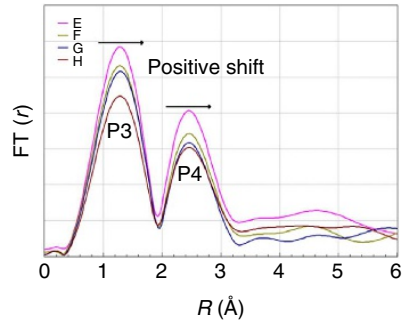

g

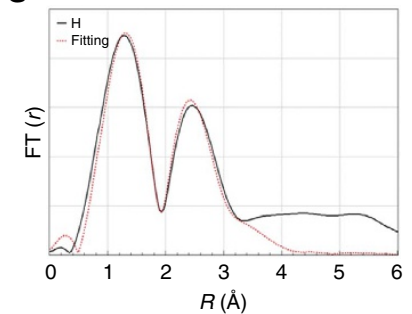

Figure 4 | Phase transformation energy profile using DFT and comparison of in situ EXAFS collected on the electrode together with their

corresponding fitted curves. (a) All energies are referenced to the total energy of the $\mathrm{CoOOH}$ ground state (state $\mathrm{C}^{\star}$ ). States $\mathrm{A}-\mathrm{C}$ stand for minima and transition structures are indicated by T1-T3; their structures are schematically shown in the insets. Asterisks denote the use of the lattice constants of $\mathrm{CoOOH}$ ground state instead of those of $\mathrm{Co}(\mathrm{OH})_{2}$. Notice that structures with and without asterisks both correspond to $\mathrm{CoOOH}$ and, therefore, contain the same amount of $\mathrm{H}$ atoms. Red, white and blue balls stand for $\mathrm{O}, \mathrm{H}$ and $\mathrm{Co}$ atoms, respectively. Total energy values for all the minima and transition states, and their corresponding optimized atomic structures are given in Supplementary Tables 3-5. (b) In situ EXAFS spectra of a whole charge/discharge cycle in a three-dimensional mode. A-H labels correspond to the positions marked in Fig. 2c. (c,d) In situ EXAFS spectra of the charge and discharge process, respectively. (e-g) The fitting results of spectra A, D and $\mathrm{H}$. The black solid lines represent the experimental curves while the red dot lines are the fitted curves.

a similar structure (Supplementary Fig. 13) to $\mathrm{NiOOH}$ that facilitates phase transformation during charge. This motif is in perfect accordance with the case of $\mathrm{Co}(\mathrm{OH})_{2} / \mathrm{CoOOH}$. When $\mathrm{Co}(\mathrm{OH})_{2}$ acts as a SC electrode, a similar battery-mimic mechanism is more favourable, as $\mathrm{Co}(\mathrm{OH})_{2}$ and $\mathrm{CoOOH}$ have similar lattice structures just like the case of $\mathrm{Ni}(\mathrm{OH})_{2} / \mathrm{NiOOH}$ to facilitate the redox process. It is Faradaic in origin, involving the passage of charge across the double layer within the change of potential. Within a certain working window, a material with more distinct oxidation states is expected to achieve higher capacitance ${ }^{1}$. In contrast, Fe oxides show poor pseudocapacitance properties $^{3,26-28}$. The dissimilarities of structural types (Supplementary Fig. 14) between Fe oxides and hydroxides pull down SC, which demands a fast phase transformation, especially for pseudocapacitance materials. The lattice structures change between different Fe phases is expected to cost too much energy due to the required massively rearrangement of atoms. In the end, it is the similarity between pristine and charged phases that originates excellent performance of $\mathrm{Co}(\mathrm{OH})_{2}$ and $\mathrm{Ni}(\mathrm{OH})_{2}$, which Fe oxides and hydroxides do not possess. The novelty and originality of our work lies right in that we successfully interpreted why $\mathrm{Co}(\mathrm{OH})_{2}$ shows distinct peaks in cyclic 
voltammetry curves and plateaus in the galvanostatic charge/ discharge, similar to the behaviour of batteries, but still enabling excellent power densities and long cycling life with considerable energy densities. The foundation of the $\mathrm{Co}(\mathrm{OH})_{2}$, synthesized through one-step method without binders, having excellent capacity and cyclability, is the intrinsic structural similarity and the battery-mimic mechanism we demonstrated, which spares unnecessary and unfavourable massive atom rearrangements, and offers practical potentials of excellent electrochemical properties. SC electrode materials use their active surface to storage energy, while battery electrode materials are bulk materials for ion intercalation/de-intercalation. From the perspective of SCs, the structural similarity between $\mathrm{Co}(\mathrm{OH})_{2}$ and $\mathrm{CoOOH}$ spares massive structural changes for this phase transformation, resulting in excellent power densities and long cycling life. From the perspective of batteries, $\mathrm{H}^{+}$intercalation/deintercalation for energy store/release (a mechanism similar to, for example, $\mathrm{Li}^{+}$intercalation/de-intercalation) is the key factor to achieve the observed high energy densities. This can benefit future supercapacitor materials designs to yield high energy densities. In particular, the transformation between $\mathrm{Co}(\mathrm{OH})_{2}$ and $\mathrm{CoOOH}$ is a good model to pursue enhanced properties and minimize drawbacks, providing a novel concept for the hybridization of SCs and batteries. For the hybrid material design, we should design materials with structural similarity between their origin and charged phases so that we can preserve the excellent properties of SCs, such as quick charge/discharge capability and high power density. On the other hand, based on this battery-mimic mechanism, we can perform some theoretical calculations to predict the maximum thickness of active materials, which can be utilized for ion intercalation/de-intercalation to enhance energy density. And of course, other factors like conductivity should be also under consideration. $\mathrm{Co}(\mathrm{OH})_{2}$ is a beacon that shows us the way to pursue the hybrid device of SCs and batteries for further developments, but there are many work to be done and new materials needs to be delicately designed for hybridization of SCs and batteries.

\section{Discussion}

The structural similarity between $\mathrm{Co}(\mathrm{OH})_{2}$ and $\mathrm{CoOOH}$ enables a battery-mimic mechanism, resulting in high specific pseudocapacitance and long cycling life. The different behaviours of ironseries elements prove this structure-to-property affinity. $\mathrm{Co}(\mathrm{OH})_{2}$ electrodes with a battery-mimic mechanism blurs therefore the distinction between SCs and batteries. At molecular level, the similarity between pristine and charged phases is ultimately responsible for the observed enhanced properties and performance. Our results may radically alter the design route of new electrodes for SC, battery and hybrid device materials. Lithiumion batteries can deliver a high energy density, but are limited by power density and cycling stability ${ }^{29,30}$. On the other hand, SCs still suffer from low energy density. Our findings pave the way for new pseudocapacitance materials with high energy density and high power density for a wide range of applications, including high-power-density batteries with considerable energy densities. Our work also sheds light on the promising future for the hybridization of SCs and batteries, with great potential for future energy storage applications.

\section{Methods}

Synthesis of $\mathrm{Co}(\mathrm{OH})_{2}$. Our previous work demonstrated that the electrodeposition is an ideal synthesis method to prepare binder-free electrode for better performance ${ }^{17}$. In this work, a CFP was used as the substrate. Before the preparation, the CFP was ultrasonically cleaned with acetone, ethanol and distilled water for $30 \mathrm{~min}$, respectively. $\mathrm{Co}(\mathrm{OH})_{2}$ thin film was electrodeposited in an aqueous solution containing $1.2 \mathrm{M} \mathrm{Co}\left(\mathrm{NO}_{3}\right)_{2}$ (analytical reagent (AR), $\geq 99.0 \%$, Xilong Chemical Co., Ltd.). The deposition process was performed at $45^{\circ} \mathrm{C}$ in a
}

conventional three-electrode electrolytic cell consisting of the CFP $\left(2 \mathrm{~cm}^{2}\right.$ in area) a platinum counter electrode $\left(2 \mathrm{~cm}^{2}\right.$ in area) and a saturated calomel electrode reference. The deposition potential was controlled at $-0.9 \mathrm{~V}$ for $60 \mathrm{~min}$. The electrodeposition process of the $\mathrm{Co}(\mathrm{OH})_{2}$ film can be expressed as follows ${ }^{31}$ :

$$
\begin{gathered}
\mathrm{NO}_{3}^{-}+7 \mathrm{H}_{2} \mathrm{O}+8 e^{-} \rightarrow \mathrm{NH}_{4}^{+}+10 \mathrm{OH}^{-} \\
\mathrm{Co}^{2+}+2 \mathrm{OH}^{-} \rightarrow \mathrm{Co}(\mathrm{OH})_{2}
\end{gathered}
$$

After the deposition, the prepared electrode was rinsed consequentially with double-distilled water and dried in vacuum oven at $60^{\circ} \mathrm{C}$ for $12 \mathrm{~h}$. The mass of the deposited $\mathrm{Co}(\mathrm{OH})_{2}$ was measured for the weight difference before and after the electrochemical deposition by means of a micro-balance (Sartorius BT125D) with an accuracy of $0.01 \mathrm{mg}$.

Physical characterization. Surface morphology of the prepared electrode, after bathing in $1 \mathrm{M} \mathrm{KOH}$ for $15 \mathrm{~min}$, and after in situ XAS measurements, was studied by using SEM (an Hitachi SU8000 scanning electron microscope operated at $2 \mathrm{kV}$ ). The detailed microstructural characterization was performed by TEM

(JEM ARM 1300S for high-resolution TEM images and Libra 200 HT Mc Cs for selected area electron diffraction pattern and bright field (BF) images). The specific area was examined by $\mathrm{N}_{2}$ adsorption measurements using an auto JW-BK132F instrument from JWGB SCI. \& TECH instruments. Fourier transform infrared spectra were obtained from Perkin Elmer Spectrum One B instrument. X-ray photoelectron spectroscopy (XPS) spectra were acquired from ESCALAB-250 instrument with a monochromatic $\mathrm{Al} \mathrm{K} \alpha$ radiation source and a hemisphere detector with an energy resolution of $0.1 \mathrm{eV}$. The $\mathrm{Co}(\mathrm{OH})_{2}$ phase was investigated using X-ray diffraction (RIGAKU D/MAX2500, $\lambda=1.54 \mathrm{~nm}$ ). For more detailed information, X-ray diffraction spectra of the prepared $\mathrm{Co}(\mathrm{OH})_{2}$ electrode was collected at Shanghai Synchrotron Radiation Facility, on BL14B1 beamline with an electron energy of $2 \mathrm{GeV}$.

Electrochemical characterization. Electrochemical measurements were carried out on a computer-controlled potentiostat (CHI660E, $\mathrm{CH}$ instrument, Shanghai) with a three-electrode electrochemical cell contain $1 \mathrm{M} \mathrm{KOH}$ aqueous solution as electrolyte. The working electrode was the $\mathrm{Co}(\mathrm{OH})_{2}$. A platinum plate and an saturated calomel electrode were used as counter electrode and reference electrode, respectively. The electrolyte $(1 \mathrm{M} \mathrm{KOH})$ was prepared from high-purity $\mathrm{KOH}$ pellets (AR, $\geq 85.0 \%$, Sinopharm Chemical Reagent Co., Ltd.) by adding $56 \mathrm{~g}$ of pellets to 11 distilled water. Before the electrochemical activity was measured, the $\mathrm{Co}(\mathrm{OH})_{2}$ was bathed in the electrolyte for $15 \mathrm{~min}$ to make $\mathrm{OH}^{-}$infiltrated in the interlayer space. The active carbon (AC) electrode was fabricated using method as follows: a mixture of AC, acetylene black, nafion (wt\%: 85:10:5) and a small amount of ethanol was prepared by stirring $6 \mathrm{~h}$ to produce a homogeneous paste. This paste was pressed onto Ni foam to produce AC electrode. A two-electrode cell configuration was used to measure the performance of an asymmetric SC in $1 \mathrm{M}$ $\mathrm{KOH}$ aqueous electrolyte solution. The working electrodes were the prepared $\mathrm{Co}(\mathrm{OH})_{2}$ and $\mathrm{AC}$ electrodes mentioned above, and they were placed together and separated by a porous non-woven cloth separator. Then cyclic voltammetry was used to investigate its activity. Its charge/discharge ability was measured by a galvanostatic test. The energy and power densities of the asymmetric capacitor were calculated as follow: $E=0.5 \times C \times V^{2}, P=I \times V / m$, where $E\left(\mathrm{Wh} \mathrm{kg}^{-1}\right)$ is the energy density and $P\left(\mathrm{~W} \mathrm{~kg}^{-1}\right)$ is the power density.

In situ Co K-edge XAS. X-ray absorption spectra were collected at Beijing Synchrotron Facility (BSRF) on beamline 1W1B. The BSRF storage ring is operated at the electron energy of $2.2 \mathrm{GeV}$ with beam current of $250 \mathrm{~mA}$. A Si (111) doublecrystal monochromator was applied. The beam size used at the sample position was about $900 \times 300 \mu \mathrm{m}^{2}$. For electrochemical experiments, no transmission data could be collected, and then fluorescence mode was used. All the data were collected at ambient temperature. Curve fitting was performed with Artemis and IFEFFIT software ${ }^{24,32}$, EXAFS curve fitting of these three spectra was carried out using both $\mathrm{Co}(\mathrm{OH})_{2}$ and $\mathrm{CoOOH}$ data as the starting parameters. Before collecting the XAS spectra, the electrode was cycled 30 times in a three-electrode cell to reach a steady state. In situ electrochemistry was performed in the self-made device (Fig. 1b) and cyclic voltammetry was used from -0.3 to $0.4 \mathrm{~V}$. To meet the condition of XAS sampling, we set the scan rate at $0.4 \mathrm{mV} \mathrm{s}^{-1}$. Spectra were acquired in QuickEXAFS (QXAS) mode. Cobalt oxide (99.995\%, Alfa Aesar, may contain up to $10 \%$ $\mathrm{Co}_{3} \mathrm{O}_{4}$ ) was purchased to be the reference.

DFT calculations. Spin-polarized DFT calculations were performed using supercell approach and the Perdew-Burke-Ernzerhof (PBE) functional ${ }^{33}$ as implemented in the Vienna ab initio simulation package (VASP, version 5.4.1) ${ }^{34}$. We treated explicitly the $\mathrm{H}(1 s), \mathrm{O}(2 s, 2 p)$ and Co $(3 s, 3 p, 3 d, 4 s)$ electrons as valence states expanded in plane waves with a cut-off energy of $520 \mathrm{eV}$, whereas the remaining electrons were replaced by PBE-based projector-augmented wave potentials ${ }^{35}$. Total energies and electron densities were computed using the DFT $+\mathrm{U}$ approach of Dudarev et al. ${ }^{36}$, in which a Hubbard U-like term describing the onsite Coulomb interactions $\left(U_{\text {eff }}=U-J\right.$, that is, the difference between the Coulomb $U$ and 
exchange $J$ parameters, hereinafter referred to as simply $U$ ) is added to the PBE functional. We used a value of $U=3.3 \mathrm{eV}$ for Co atoms, which was obtained by Ceder $e t a l .{ }^{37}$ on the basis of oxide formation energies and agrees well with other $U$ values recently proposed to describe different cobalt oxide and hydroxide phases $^{38}$, as well as $\mathrm{CoOOH}$ (ref. 39) and Co-doped $\mathrm{NiOOH}$ (ref. 40) surfaces. We used a unit cell containing two formula units $\left(\mathrm{Co}_{2} \mathrm{O}_{4} \mathrm{H}_{2}\right)$. Equilibrium lattice constants were computed allowing the atomic positions, lattice vectors and cell shape to relax with a residual force threshold of $0.02 \mathrm{eV}^{-1}$ and using a Monkhorst - Pack grid with $8 \times 8 \times 2 k$-point sampling. These computational settings guarantee a tight convergence in equilibrium distances (better than $0.001 \AA$ ). Transition structures for the considered phase transformations were located by using the nudged elastic band algorithm ${ }^{41}$ using five images along each pathway. The optimized lattice constants were used for all of the nudged elastic band calculations, which were performed at constant volume.

Surface calculations were performed using stoichiometric slab models cut along five low-index surface directions of $\beta-\mathrm{Co}(\mathrm{OH})_{2}$ (space group P-3m1): (0001); $(10-10) ;(11-20) ;(01-12)$; and (10-14). Each slab model contained four to six atomic $\mathrm{Co}(\mathrm{OH})_{2}$ layers, with $15 \AA$ of vacuum between slabs and centre layers fixed to their bulk PBE + U-optimal positions, leaving at least two layers relaxed on both sides of the slab with a residual force threshold of $0.03 \mathrm{eV} \AA^{-1}$. Supplementary Fig. 3 shows the side and top views of the optimized geometries. Calculations were carried out using $800 \mathrm{eV}$ plane-wave cut-off and $k$-point meshes of $12 \times 12 \times 1$, $10 \times 8 \times 1,14 \times 12 \times 1,8 \times 4 \times 1$ and $12 \times 4 \times 1$ for (0001), $(10-10),(11-20)$, (01-12) and (10-14) surfaces, respectively. This set-up ensures a tight convergence in energies of at least $3 \mathrm{meV}$ per atom. The surface energy of each slab of given area $2 A$ and free energy $G_{\text {slab }}$ was computed as $\gamma=\left(G_{\text {slab }}-N g_{\text {bulk }}\right) / 2 A$, where $N$ is the number of $\mathrm{Co}(\mathrm{OH})_{2}$ formula units in the slab and $g_{\text {bulk }}$ is the free energy per formula unit of the ground state bulk $\mathrm{Co}(\mathrm{OH})_{2}$ (space group $\mathrm{C2}$ ). We neglected the zero point energy and entropy corrections for $G_{\text {slab }}$ and $g_{\text {bulk, }}$, which then become equal to the DFT total energies, $E_{\text {slab }}$ and $E_{\text {bulk }}$, respectively.

Data availability. The data that support the findings of this study are available from the corresponding authors on request.

\section{References}

1. Conway, B. E. Electrochemical Supercapacitors: scientific Fundamentals and Technological Applications (Plenum, 1999).

2. Simon, P. \& Gogotsi, Y. Materials for electrochemical capacitors. Nat. Mater. 7, 845-854 (2008).

3. Wang, G., Zhang, L. \& Zhang, J. A review of electrode materials for electrochemical supercapacitors. Chem. Soc. Rev. 41, 797-828 (2012).

4. Arico, A. S., Bruce, P., Scrosati, B., Tarascon, J. M. \& Van Schalkwijk, W. Nanostructured materials for advanced energy conversion and storage devices. Nat. Mater. 4, 366-377 (2005).

5. Steele, B. C. H. \& Heinzel, A. Materials for fuel-cell technologies. Nature 414, 345-352 (2001).

6. Cheng, F. Y., Liang, J., Tao, Z. L. \& Chen, J. Functional materials for rechargeable batteries. Adv. Mater. 23, 1695-1715 (2011).

7. Goodenough, J. B. \& Park, K. S. The Li-ion rechargeable battery: a perspective. J. Am. Chem. Soc. 135, 1167-1176 (2013).

8. Chen, J. \& Cheng, F. Y. Combination of lightweight elements and nanostructured materials for batteries. Acc. Chem. Res. 42, 713-723 (2009).

9. Li, H. B. et al. Amorphous nickel hydroxide nanospheres with ultrahigh capacitance and energy density as electrochemical pseudocapacitor materials. Nat. Commun. 4, 1894 (2013).

10. Cao, L., Xu, F., Liang, Y. Y. \& Li, H. L. Preparation of the novel nanocomposite $\mathrm{Co}(\mathrm{OH}) 2$ /ultra-stable $\mathrm{Y}$ zeolite and its application as a supercapacitor with high energy density. Adv. Mater. 16, 1853-1857 (2004).

11. Gao, S. et al. Ultrahigh energy density realized by a single-layer beta- $\mathrm{Co}(\mathrm{OH}) 2$ all-solid-state asymmetric supercapacitor. Angew. Chem. Int. Ed. 53, 12789-12793 (2014)

12. Li, B. et al. Improved performances of beta- $\mathrm{Ni}(\mathrm{OH}) 2 @$ reduced-graphene-oxide in Ni-MH and Li-ion batteries. Chem. Commun. 47, 3159-3161 (2011).

13. Simon, P., Gogotsi, Y. \& Dunn, B. Where do batteries end and supercapacitors begin? Science 343, 1210-1211 (2014).

14. Huang, L. et al. Nickel-cobalt hydroxide nanosheets coated on NiCo2O4 nanowires grown on carbon fiber paper for high-performance pseudocapacitors. Nano Lett. 13, 3135-3139 (2013).

15. Liu, Z., Ma, R., Osada, M., Takada, K. \& Sasaki, T. Selective and controlled synthesis of alpha- and beta-cobalt hydroxides in highly developed hexagonal platelets. J. Am. Chem. Soc. 127, 13869-13874 (2005).

16. Kong, L.B., Lang, J.W., Liu, M., Luo, Y.C. \& Kang, L. Facile approach to prepare loose-packed cobalt hydroxide nano-flakes materials for electrochemical capacitors. J. Power Sources 194, 1194-1201 (2009).

17. Zhao, C. et al. Synthesis of $\mathrm{Co}(\mathrm{OH}) 2 /$ graphene/ $\mathrm{Ni}$ foam nano-electrodes with excellent pseudocapacitive behavior and high cycling stability for supercapacitors. Int. J. Hydrogen Energy 37, 11846-11852 (2012).
18. Gaunand, A. \& Lim, W. L. From amorphous precipitates to sub-micronic crystalline platelets of $\mathrm{Co}(\mathrm{OH})(2)$ : a kinetic study of the transformation process. Powder Technol. 128, 332-337 (2002).

19. Ghodbane, O., Pascal, J. L. \& Favier, F. Microstructural effects on chargestorage properties in $\mathrm{MnO} 2$-based electrochemical supercapacitors. ACS Appl. Mater. Interfaces 1, 1130-1139 (2009).

20. Chang, J.-K., Wu, C.-M. \& Sun, I. W. Nano-architectured $\mathrm{Co}(\mathrm{OH}) 2$ electrodes constructed using an easily-manipulated electrochemical protocol for highperformance energy storage applications. J. Mater. Chem. 20, 3729 (2010).

21. Wang, L., Dong, Z. H., Wang, Z. G., Zhang, F. X. \& Jin, J. Layered -Co(OH)2 nanocones as electrode materials for pseudocapacitors: understanding the effect of interlayer space on electrochemical activity. Adv. Func. Mater. 23, 2758-2764 (2013).

22. Zhao, C. M. et al. Ultrahigh capacitive performance from both $\mathrm{Co}(\mathrm{OH})(2) /$ graphene electrode and K3Fe(CN)(6) electrolyte. Sci. Rep. 3, 2986 (2013).

23. Pantelouris, A., Modrow, H., Pantelouris, M., Hormes, J. \& Reinen, D. The influence of coordination geometry and valency on the K-edge absorption near edge spectra of selected chromium compounds. Chem. Phys. 300, 13-22 (2004).

24. Rehr, J. J. \& Albers, R. C. Theoretical approaches to X-ray absorption fine structure. Rev. Mod. Phys. 72, 621-654 (2000).

25. Wang, H. L., Casalongue, H. S., Liang, Y. Y. \& Dai, H. J. Ni(OH)(2) nanoplates grown on graphene as advanced electrochemical pseudocapacitor materials. J. Am. Chem. Soc. 132, 7472-7477 (2010).

26. Wasiński, K., Walkowiak, M., Półrolniczak, P. \& Lota, G. Capacitance of $\mathrm{Fe} 3 \mathrm{O} 4 / \mathrm{rGO}$ nanocomposites in an aqueous hybrid electrochemical storage device. J. Power Sources 293, 42-50 (2015).

27. Qu, Q., Yang, S. \& Feng, X. 2D sandwich-like sheets of iron oxide grown on graphene as high energy anode material for supercapacitors. Adv. Mater. 23, 5574-5580 (2011).

28. Li, Y. et al. The impact of morphologies and electrolyte solutions on the supercapacitive behavior for $\mathrm{Fe} 2 \mathrm{O} 3$ and the charge storage mechanism. Electrochim. Acta 178, 171-178 (2015).

29. Tarascon, J. M. \& Armand, M. Issues and challenges facing rechargeable lithium batteries. Nature 414, 359-367 (2001).

30. Choi, N. S. et al. Challenges facing lithium batteries and electrical double-layer capacitors. Angew. Chem. Int. Ed. 51, 9994-10024 (2012).

31. Zhao, T., Jiang, H. \& Ma, J. Surfactant-assisted electrochemical deposition of $\alpha$-cobalt hydroxide for supercapacitors. J. Power Sources 196, 860-864 (2011).

32. Newville, M. EXAFS analysis using FEFF and FEFFIT. J. Synchrotron Radiat. 8, 96-100 (2001).

33. Perdew, J. P. et al. Erratum: restoring the density-gradient expansion for exchange in solids and surfaces. Phys. Rev. Lett. 102, 039902 (2009).

34. Kresse, G. \& Furthmüller, J. Efficient iterative schemes for ab initio total-energy calculations using a plane-wave basis set. Phys. Rev. B 54, 11169-11186 (1996).

35. Kresse, G. \& Joubert, D. From ultrasoft pseudopotentials to the projector augmented-wave method. Phys. Rev. B 59, 1758-1775 (1999).

36. Dudarev, S. L., Botton, G. A., Savrasov, S. Y., Humphreys, C. J., A. \& Sutton, A. P. Electron-energy-loss spectra and the structural stability of nickel oxide: an LSDA + U study. Phys. Rev. B 57, 1505-1509 (1998).

37. Wang, L., Maxisch, T. \& Ceder, G. Oxidation energies of transition metal oxides within the GGA + U framework. Phys. Rev. B 73, 195107 (2006).

38. Chen, J. \& Selloni, A. First principles study of cobalt (Hydr)oxides under electrochemical conditions. J. Phys. Chem. C 117, 20002-20006 (2013).

39. Garcia-Mota, M. et al. Importance of correlation in determining electrocatalytic oxygen evolution activity on cobalt oxides. J. Phys. Chem. C 116, 21077-21082 (2012).

40. Costanzo, F. Effect of doping b-NiOOH with $\mathrm{Co}$ on the catalytic oxidation of water: DFT + U calculations. Phys. Chem. Chem. Phys. 18, 7490 (2016).

41. Jónsson, H., Mills, G. \& Jacobsen, K. W. Classical and Quantum Dynamics in Condensed Phase Simulations (World Scientific, 1998).

\section{Acknowledgements}

This research is supported by the National Natural Science Foundation of China (No. 51372095), Special Funding for Academic Leaders (No. 419080500630) at Jilin University, and Korea Basic Science Institute grant (KBSI, T37210) to J.-G.K. W.Z. acknowledges the KBSI under the R\&D programme (No. D36700) supervised by the Ministry of Science, ICT and Future Planning Korea. Beijing Synchrotron Radiation Facility (BSRF) is greatly acknowledged for providing us the beam time on beamline 1W1B for the XAS measurement, and Shanghai Synchrotron Radiation Facility (SSRF) on beamline BL14B1 for the X-ray diffraction measurement. J.C. acknowledges support by The Royal Society through the Newton Alumnus scheme. We are also grateful for computer resources to the Barcelona Supercomputer Center and the Arina cluster (Universidad del País Vasco/Euskal Herriko Unibertsitatea). We express our sincere thanks to Sibing Wang, Haifeng Zhao, Xiyang Wang, Haoxiang Wang and Dong Wang for their stimulating discussions and help. 


\section{Author contributions}

W.Z., W.T.Z. and T.D. conceived and coordinated the study and led writing of the manuscript; T.D. conducted the XAS and X-ray diffraction analysis; O.A. and J.C. contributed the theoretical calculations and analysis; J.-G.K., W.Z. and S.J.Y. contributed the microscopy and microanalysis; T.D. and J.W. and contributed the electrochemical test. All co-authors commented on the manuscript.

\section{Additional information}

Supplementary Information accompanies this paper at http://www.nature.com/ naturecommunications

Competing interests: The authors declare no competing financial interests.

Reprints and permission information is available online at http://npg.nature.com/ reprintsandpermissions/
How to cite this article: Deng, T. et al. Atomic-level energy storage mechanism of cobalt hydroxide electrode for pseudocapacitors. Nat. Commun. 8, 15194 doi: $10.1038 /$ ncomms15194 (2017)

Publisher's note: Springer Nature remains neutral with regard to jurisdictional claims in published maps and institutional affiliations.

(C) This work is licensed under a Creative Commons Attribution 4.0 International License. The images or other third party material in this article are included in the article's Creative Commons license, unless indicated otherwise in the credit line; if the material is not included under the Creative Commons license, users will need to obtain permission from the license holder to reproduce the material. To view a copy of this license, visit http://creativecommons.org/licenses/by/4.0/

(C) The Author(s) 2017 